\title{
Brimonidine Tartrate
}

National Cancer Institute

\section{Source}

National Cancer Institute. Brimonidine Tartrate. NCI Thesaurus. Code C47419.

The tartrate salt form of brimonidine, an imidazole derivative and a selective alpha-2

adrenergic receptor agonist. Upon ocular administration, brimonidine tartrate acts on the blood vessels causing them to constrict which leads to a decrease in the production of aqueous humor. Brimonidine tartrate also enhances the outflow of aqueous humor. This drug is used in the treatment of glaucoma to reduce intraocular pressure. 